\title{
The combination of multiple materials in the creation of an artificial anterior chest cage after extensive demolition for recurrent chondrosarcoma
}

Gaetano Rocco, MD, FRCS (Ed), ${ }^{\text {a }}$ Flavio Fazioli, MD, ${ }^{\text {b }}$ Francesco Scognamiglio, MD, ${ }^{\text {a }}$ Valerio Parisi, MD, ${ }^{\text {b }}$ Carmine La Manna, MD, ${ }^{\text {a }}$ Antonello La Rocca, MD, ${ }^{a}$ Rocco Cerra, MD, ${ }^{\mathrm{b}}$ Rosanna Accardo, MD, and Elisabetta De Lutio, MD, ${ }^{\mathrm{c}}$ Naples, Italy

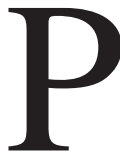

rimary sarcomas represent a serious oncologic challenge because, at times, their surgical removal creates large defects requiring covering with several autologous, homologous, or prosthetic materials used in 1 or 2 graft combinations. ${ }^{1-4}$ Although the experience is more limited, relapsing sarcomas often call for even more extensive demolitions of the chest wall and difficult reconstructive efforts. $^{5}$

From the Division of Thoracic Surgery ${ }^{\mathrm{a}}$ and Departments of Surgery ${ }^{\mathrm{b}}$, and Radiology, ${ }^{\mathrm{c}}$ National Cancer Institute, Pascale Foundation, Naples, Italy,

Received for publication Oct 29, 2006; accepted for publication Nov 28, 2006.

Address for reprints: Gaetano Rocco, MD, FRCS (Ed), FECTS, Division of Thoracic Surgery, National Cancer Institute, Pascale Foundation, Via M Semmola, 81, 80131 Naples, Italy (E-mail: Gaetano.Rocco@ btopenworld.com).

J Thorac Cardiovasc Surg 2007;133:1112-4

$0022-5223 / \$ 32.00$

Copyright $\odot 2007$ by The American Association for Thoracic Surgery doi:10.1016/j.jtcvs.2006.11.045

\section{Clinical Summary}

A 64-year-old man presented in January 2006 with a massive locoregional recurrence of a chest wall chondrosarcoma (Figure 1) originally resected 10 months earlier, on tumor-free margins, by removing the anterior right-sided ribs III to VI and covering the chest wall defect with Marlex (Bard, Cranston, RI) mesh reinforced by a latissimus dorsi flap.

An extensive demolition of the anterior chest wall was performed by dividing the manubrium and the anterior ribs from ribs II to VIII along the hemiclavicular line and the anterior axillary line of the left and right sides, respectively. The anterior pericardium, the central tendon, and part of the muscle component of the diaphragm were also removed. Both rectus abdominis muscles were divided in their most cranial aspect. The tumor was noted to infiltrate the liver capsule and marginally the right middle lobe of the lung. The involved areas were resected, and the specimen was removed on tumor-free margins.

The reconstructive stage of the operation (Figure 2) included the replacement of the diaphragm by a polytetrafluoroethylene patch (Gore-Tex; WL Gore and Associates, Flagstaff, Ariz) through which an omentum flap was transposed into the chest. The omentum was placed to protect the heart and provide a

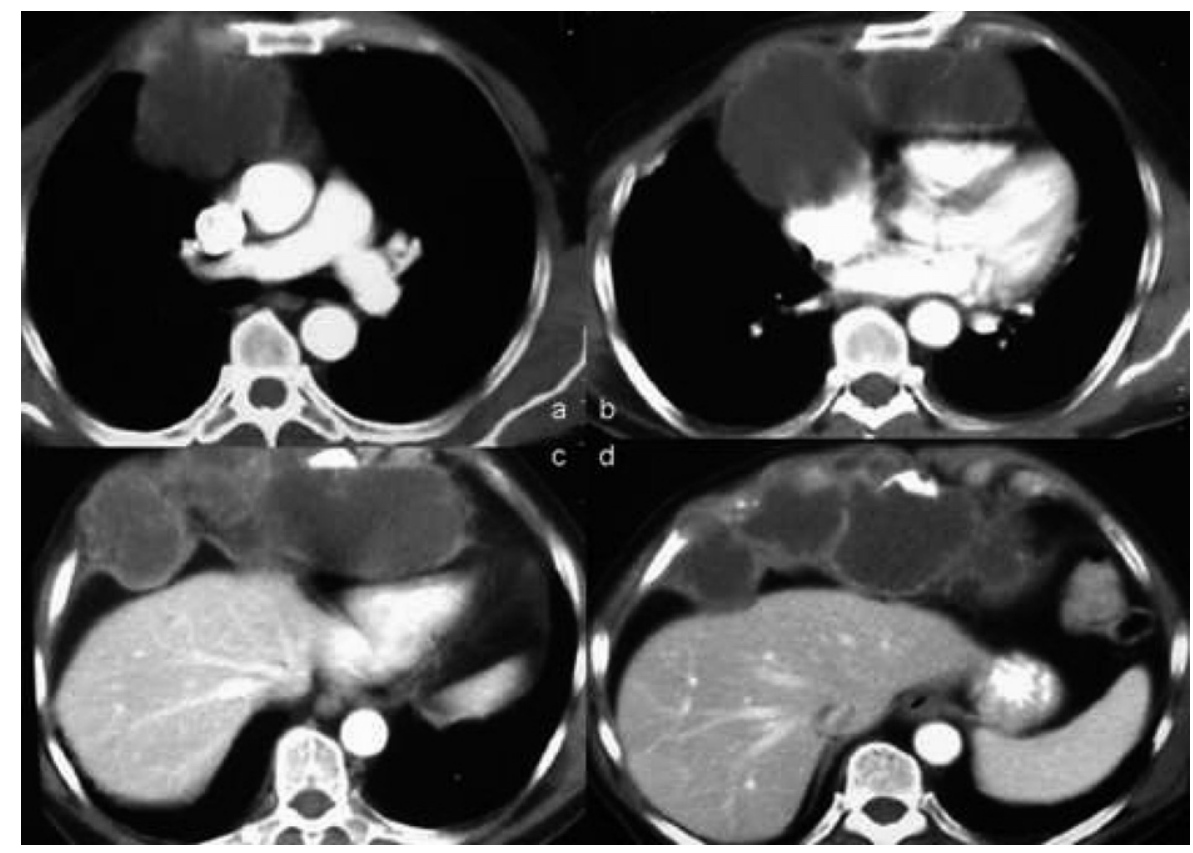

Figure 1. Chest computed tomography showing the extent of the recurrence from chondrosarcoma at different levels.

1112 The Journal of Thoracic and Cardiovascular Surgery • April 2007 


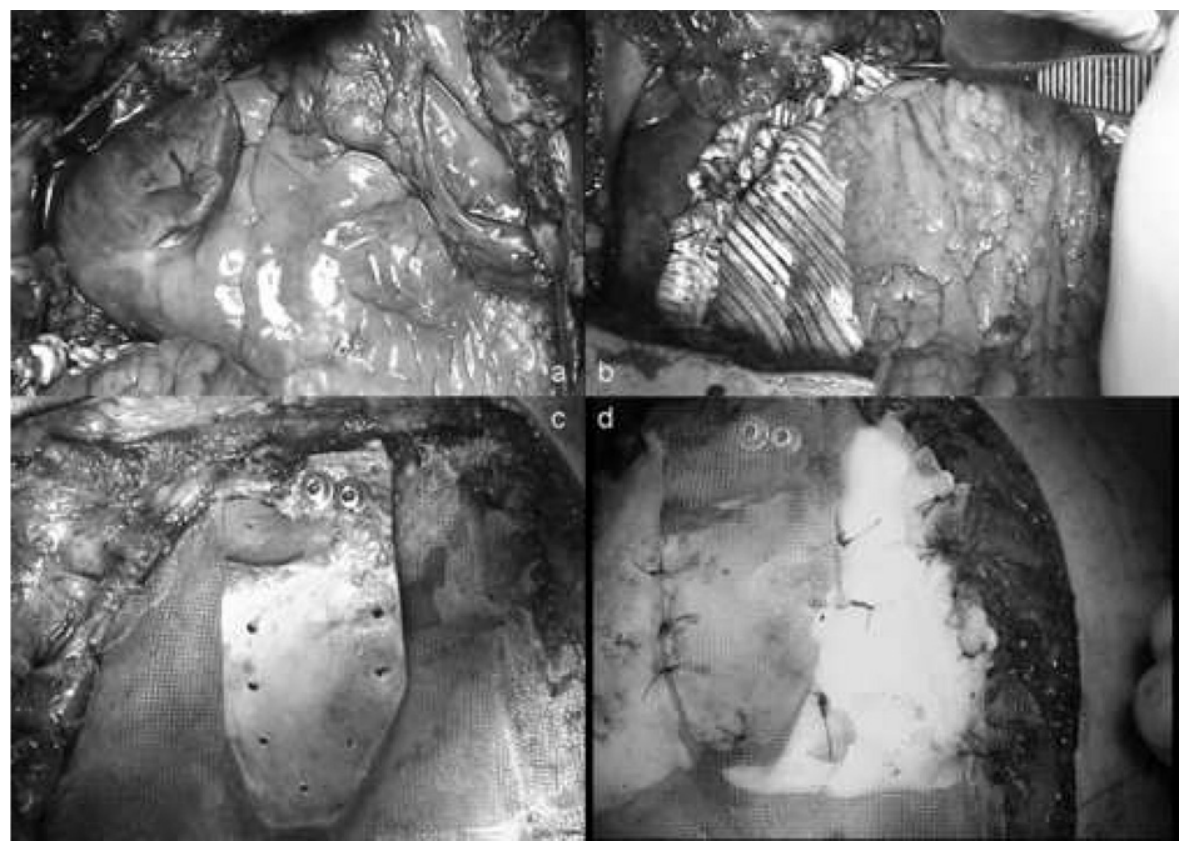

Figure 2. Intraoperative sequence. a, Intrathoracic view at the end of the resection. b, Completion of the diaphragmatic patch and omental transfer to protect the mediastinum. c, Neosternum in place. $d$, Completion of the anterior chest cage reconstruction.

vascularized bed for a cryopreserved iliac crest homograft that was shaped to fit in the sternal position by securing it to the residual part of the host manubrium. Two methyl-methacrylate sandwiches (Simplex, Stryker, Mawhaw, NJ) were then anchored medially to the bone homograft by interrupted nonabsorbable sutures, laterally to the remaining rib segments, and caudally to the prosthetic diaphragmatic patch to configure the new anterior chest wall ensemble. In addition, a myocutaneous pectoralis major flap was rotated medially to cover the neosternum superficially, thereby requiring an isolated skin graft from the left inguinal region to cover the cutaneous defect in the deltoid-pectoral area.

The patient was successfully discharged 2 weeks after surgery with no immunosuppressants other than low-dose steroids (Figure

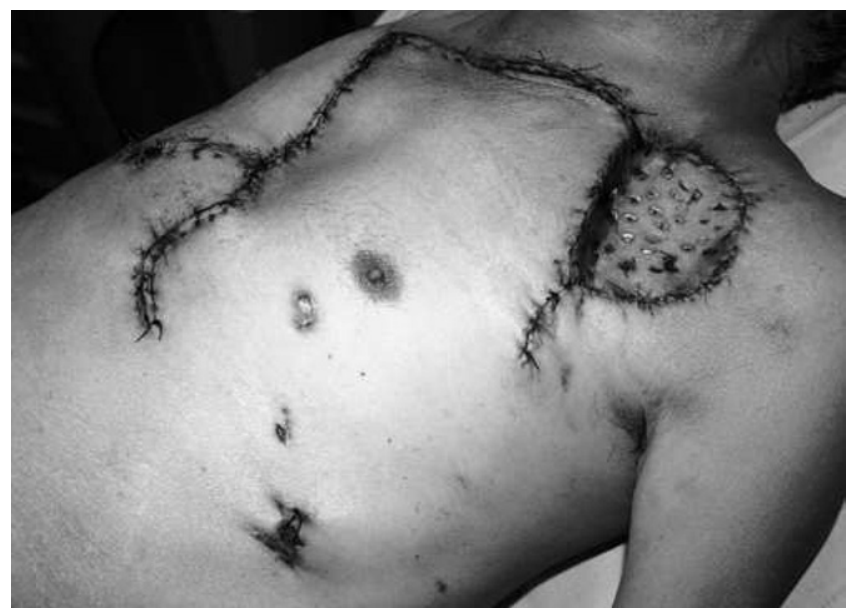

Figure 3. Patient's picture at discharge.
3). He went back to his normal activities, including amateur swimming. At more than 10 months from surgery, the patient is alive and well, albeit with isolated liver and lung recurrences for which he is undergoing chemotherapy.

\section{Discussion}

The primary aim of a complex reconstructive strategy after extensive chest wall demolition is to restore the geometric configuration of the chest cage using materials that could guarantee protection of the intrathoracic organs and structural and functional integrity. ${ }^{2,3}$ This is especially true after removal of the sternum and contiguous ribs, ${ }^{2}$ thus, in our case, the choice of the iliac crest homograft for sternal replacement and the lateral methylmethacrylate sandwiches to replace the bony chest wall to reproduce, as closely as possible, the anterior chest wall geometric configuration. The "flail chest" physiology was also avoided by recreating the costophrenic recesses by suturing the diaphragmatic patch to the remaining diaphragmatic muscle and the caudal portions of the methyl-methacrylate sandwiches. ${ }^{3}$

We believe that the uniqueness of this case of recurrent chest wall tumor resides in the simultaneous use in the same patient of almost all available reconstructive materials and techniques enabling the surgeon to perform extended demolitions and replace elements of the crucial structural frame of the chest cage to warrant margin-free oncologic resections and provide an acceptable functional outcome.

\section{References}

1. Lequaglie C, Brega Massone P, Giudice G, Conti B. Gold standard for sternectomies and plastic reconstructions after resection of primary or secondary sternal neoplasms. Ann Surg Oncol. 2002;9:472-9. 
2. Deschamps C, Tirnaksiz BM, Darbandi R, Trastek VF, Allen MS, Miller DL, et al. Early and long term results of prosthetic chest wall reconstruction. J Thorac Cardiovasc Surg. 1999;117:588-92.

3. Weyant MJ, Bains MSD, Venkatraman E, Downey RJ, Park BJ, Flores RM, et al. Results of chest wall resection and reconstruction with and without rigid prosthesis. Ann Thorac Surg. 2006;81:279-85.
4. Garcia-Tutor E, Yeste L, Murillo J, Aubà C, Sanjulian M, Torre W. Chest wall reconstruction using iliac bone allografts and muscle flaps. Ann Plast Surg. 2004;52:54-60.

5. Fisher S, de Perrot M, Sekine Y, Keshavjee S. Long term survival after multiple resections of a fibrosarcoma involving the lung and the chest wall. Eur J Cardiothorac Surg. 2001;20:421-3.

\section{Mediastinal mature teratoma with complex rupture into the pleura, lung, and bronchus complicated with mycoplasma pneumonia}

Ching-Wen Yu, MD, ${ }^{a}$ Ming-Jang Hsieh, MD, ${ }^{\mathrm{b}}$ Kao-Pin Hwang, MD, ${ }^{c}$ Chung-Cheng Huang, MD, ${ }^{d}$ Shu-Hang Ng, MD, ${ }^{d}$ and Sheung-Fat Ko, MD, ${ }^{d}$ Kaohsiung, Taiwan

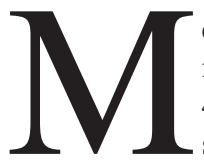

ediastinal mature teratoma (MMT) is an uncommon neoplasm in children, accounting for only $4.3 \%$ of all germ cell tumors. ${ }^{1-3}$ It may cause stridor and dyspnea by direct tracheal compression in children $<2$ years old but is rarely symptomatic in later childhood. ${ }^{1}$ Occasionally, MMT may rupture into the pleural cavity, pericardium, lung, and tracheobronchial tree, leading to protean clinical presentations. ${ }^{1-5}$ To our knowledge, cystic MMT with complex rupture into the pleura, lung, and bronchus complicated with mycoplasma pneumonia has not previously been described. We report such a rare case in a 13-year-old boy with an emphasis on the perplexing clinical and computed tomographic (CT) features and the importance of early surgical treatment.

\section{Clinical Summary}

A 13-year-old boy suffered from severe paroxysmal cough with brownish fluid expectorant and mild fever for 10 days. He visited a community hospital where the chest radiograph revealed left lower lung consolidation with effusion, and CT suggested pneumonia with necrotic changes and empyema. $\mathrm{He}$ was treated for lobar pneumonia. Despite antibiotic treatment, cough and fever persisted. He was then referred to our hospital. Physical examination revealed an acutely ill-looking patient with fever $\left(39^{\circ} \mathrm{C}\right)$ and decreased but coarse breathing sounds over the left lower chest. Abnormal laboratory data included a white blood cell count of $12,300 / \mathrm{mm}^{3}$ with $77 \%$ polymorphonuclear leukocytes and elevated C-reactive protein (129 mg/L). Serum cold-agglutinin titer of 1:32 and positive mycoplasma antibody tests (immunoglobulin $\mathrm{G}=21 \mathrm{BU} / \mathrm{mL}$, immunoglobulin $\mathrm{M}=28.1 \mathrm{BU} / \mathrm{mL}$; normal $<10 \mathrm{BU} / \mathrm{mL}$ ) were also noted. Chest radiograph showed left lower lung opacity and left pleural effusion. Chest tapping harvested $75 \mathrm{~mL}$ mildly brownish fluid with slight turbidity but culture was negative. Mycoplasma pneumonia was suspected. After 1-week antibiotic treatment, the fever subsided. However, severe paroxysmal cough with intermittent brownish fluid expectorant was still noted. Follow-up radiograph revealed persistent left lower lung opacity. Chest CT (Figure 1) revealed a heterogeneous, multiloculated, cystic mass in the left anterior mediastinum with a small fatty focus, highly suggestive of MMT. Perforation into the adjacent pleural cavity, lingular segment, and bronchus and focal oblit-
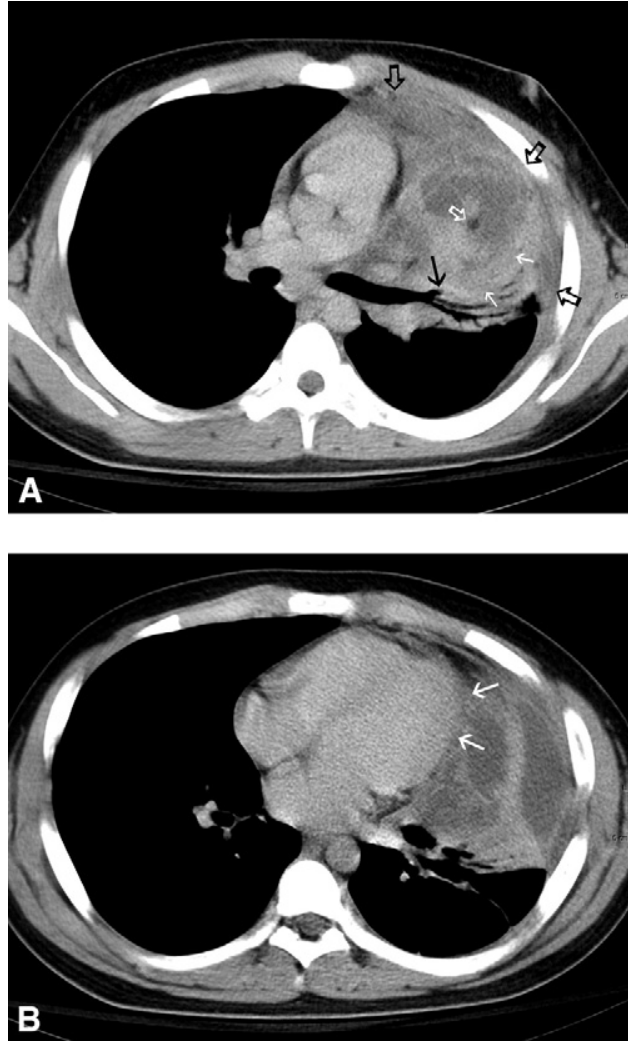

Figure 1. A, Axial contrast-enhanced CT showing a heterogeneous, multiloculated, cystic mass in the left anterior mediastinum with a small fatty focus (open white arrow) perforating into the pleura (black open arrows), lingular lobe (small white arrows), and lingular bronchus (black arrow). B, CT at the midventricular level showing obliteration of the pericardial fat (large white arrows), suggesting close attachment of the pericardium by the tumor. Minimal pericardial effusion is also noted.

eration of adjacent pericardial fat were also demonstrated. Serum alpha-fetoprotein, beta-human chorionic gonadotrophin, 